\title{
Biology begins to tangle with quantum computing
}

\author{
Quantum computing promises plenty, such as how it can massively accelerate some bioinformatics calculations.
}

\author{
Vivien Marx
}

$(2$ uantum computing is a red-hot field. Computer scientist Scott Aronson of the University of Texas at Austin points to a string of assertions about how quantum computers "will soon leave the Earth's fastest supercomputers in the dust." Researchers can find a cornucopia of funding in quantum computing. In its web portal, the large-scale EU Quantum Flagship lists funding projects and describes its intent to "kick-start a competitive European industry" in quantum technologies. A transnational network of European research funders in quantum technologies launched the QuantERA initiative, which has funding resources at https://www. quantera.eu/calls-for-proposals/call-2021. The US Congress passed the National Quantum Initiative Act, which puts in place the National Quantum Initiative Program "to accelerate the development of quantum information science and technology." Aligned with this act, the US National Science Foundation has funded three Quantum Leap Challenge Institutes, and more awards are forthcoming. Quantum Leap is about exploiting quantum mechanics to observe, manipulate and control the behavior of particles and energy at atomic and subatomic scales to lead to "next-generation technologies for sensing, computing, modeling and communicating."

Excitement in science, especially when coupled to funding opportunities, is welcome. Some biomedical scientists have begun to look at how quantum computing might help them develop and apply algorithms and machine learning approaches in many areas of biology, such as protein design ${ }^{2-5}$. When biologists use this technology, they will want to know what's happening under the hood. And consider when classical computers serve them well. "As neat as computers are, they're basically just very fast machines for doing arithmetic," says Vikram Mulligan, a researcher in systems biology at the non-profit Flatiron Institute in New York City. Computer-generated movies, video games, conversations with people hundreds of kilometers away-they all come down to



Digital bits are either 1 or 0 ; quantum bits, or qubits, can be both 1 and 0 : they can have a superposition of states. Welcome to quantum computing. Credit: M. Garlick/Science Photo Library

a series of many mathematical operations done very quickly. "Quantum computers are actually pretty slow and lousy when it comes to arithmetic," he says, but blazingly fast at other tasks.

\section{Supremacy, advantage}

Google, Honeywell, IBM and Microsoft, among others, are building quantum computing systems and demonstrating their prowess. Many smaller quantum computing companies develop products and services, including hardware and hardware components, software tools, simulation options and cloud-based quantum computing services. Some of the companies include Cambridge Quantum Computing, ColdQuanta, D-Wave Systems, IonQ, PsiQuantum, Rigetti Computing, Silicon Quantum Computing, Strangeworks, Xanadu Quantum Technologies and Zapata Computing.
In 2019, John Martinis from the University of California Santa Barbara and Google AI Quantum-he has since left Google-and his colleagues presented quantum hardware that uses a processor called Sycamore and, with academic collaborators, evaluated its performance ${ }^{6}$. The processor took 200 seconds for a task related to random number generation that, according to the team, would take a state-of-the-art classical supercomputer around 10,000 years. "Quantum processors have thus reached the regime of quantum supremacy," note the authors. That's the point at which quantum computers do what classical computers cannot, at least not in a reasonable amount of time.

John Preskill, the theoretical physicist at the California Institute of Technology who coined the term 'quantum supremacy', notes that it is associated with the "repugnant political stance" of white supremacy and 
that 'quantum supremacy' "exacerbates the already overhyped reporting on the status of quantum technology." He writes that he anticipated the second issue but failed to anticipate the first. "I expect that quantum computers will have a transformative effect on society, but this may still be decades away," notes Preskill. Some-for example, Denise Ruffner, who manages business development at the quantum computing company IonQ-prefer the term "quantum advantage" but Preskill has pointed out that 'advantage' is more like winning "by a nose." In principle, he writes, a quantum computer's speed "vastly exceeds" that of classical computers.

"Quantum supremacy is not nearly as interesting a concept as it sounds," says Lawrence Hunter from the University of Colorado School of Medicine, who works at the interface between computing and biomedicine. It just means "a workable quantum computer," he says. "The controversy comes down to the question of whether quantum computers have usefully produced an exponential speedup of anything yet." In his view, to date, quantum computing has been useful in cryptography and in simulating quantum chemistry, in which quantum mechanics is applied to aspects such as how molecules move. "Practical applications beyond those narrow areas are coming soon, and biological sequence analysis might be one of the next significant application areas," says Hunter.

Charlotte Deane, a researcher at the University of Oxford, co-authored a review ${ }^{2}$ on the prospects of quantum computing in computational molecular biology. It led a number of companies to knock at her door. Biomedical researchers should think of quantum computing as "a technology that is coming but not ready yet," says Deane. The next few years will reveal "what problems it will help solve and where it will really increase our understanding." She sees "incredible potential in the long term"-for example, in simulating molecular processes such as photosynthesis or catalysis of nitrogen fixation.

"There's a lot of buzz about quantum computing," says Yale University researcher Mark Gerstein, whose projects traverse biology and informatics. Enthusiasm among his colleagues about the prospects of quantum computing is especially high in the physical sciences, and interest is growing in computational biology and biology more generally.

When Mulligan and colleagues at several institutions posted a preprint ${ }^{3}$ about how they designed peptides on a quantum computer, it led to many conversations with companies that make quantum computing

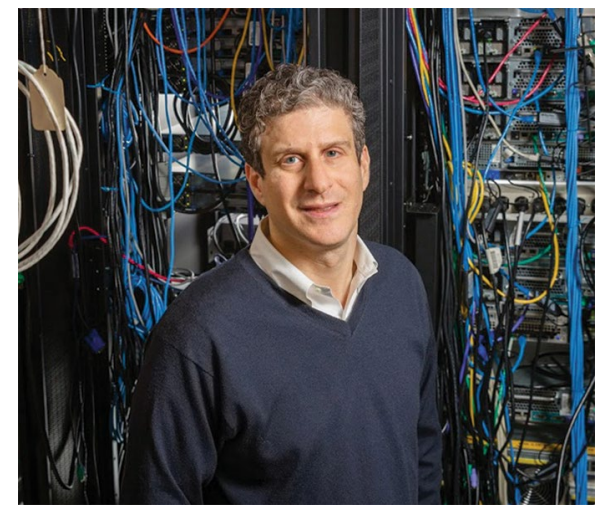

"There's a lot of buzz about quantum computing," says Yale University researcher Mark Gerstein. Enthusiasm is high in the physical sciences and interest is growing in computational biology and biology more generally. Credit: Yale Univ.

hardware and with researchers developing tools either for mapping problems to quantum hardware or for simulating quantum computers using ordinary, classical computers. "There's understandably still a fair bit of skepticism in the scientific community about any claim made about quantum computing, too, which is fair," says Mulligan. "While the potential for quantum computing is enormous, we're still in the very early days."

Research plans in quantum computing include how to apply it for combinatorial optimization and machine learning in the life sciences, says Thomas Lengauer from the Max Planck Institute for Informatics. $\mathrm{He}$ is not involved in quantum computing research but stays abreast of the area. Quantum computing applications, he says, reach well beyond the life sciences to all facets of data-driven science.

Gerstein co-authored a paper ${ }^{4}$ that grew from a series of discussions at the National Institute of Mental Health (NIMH), part of the US National Institutes of Health (NIH). It's part of the NIH's way of exploring how to support biologists interested and involved in quantum computing, he says. The wider neuroscience community, for example, is interested in how quantum approaches can be applied to deep learning and machine learning.

The NIH sessions brought together researchers in quantum computing, computational biology, neuroscience and genomics. The NIMH organizers were Geetha Senthil and her colleague Thomas Lehner, now with New York Genome Center. As Senthil says, the idea that quantum phenomena can be used to computationally solve complex problems in chemical and physical sciences "evoked curiosity in us" to explore how quantum computing might be applied in biomedicine, "especially in the light of growing challenges associated with big data and multidimensional analysis." Beyond her role at NIMH, Senthil is an NIH representative on the Subcommittee on Quantum Information Sciences (SCQIS) of the National Science and Technology Council, which coordinates federal research policy; is involved with The White House Office of Science and Technology Policy; and co-chairs an SCQIS working group set up to link biomedical researchers to quantum technologies and quantum computing. Other NIH institutes, too, she says, support biological research with advanced computing, including quantum approaches. She points to a National Institute for Drug Abuse grant related to multimodal data analysis in addiction research. 'Quantum' is generally seen more frequently in the context of physical sciences, and biologists have been less exposed to quantum science developments, says Senthil; most quantum technology applications won't be in biomedical domains. But for biology she sees practical applications in quantum sensing and quantum simulation technologies, and those would include imaging, spectroscopy, microscopy and studies of protein and molecular dynamics.

One day, says Gerstein, the NIH might operate a large quantum computer that a lab could book time on for a slice of a team's calculations. Gerstein has a supplemental grant to explore quantum computing. At the moment, the areas he sees most amenable to quantum computing are calculations in drug design and structural biology.

\section{Cats in boxes}

According to quantum mechanics, a bit of matter such as an electron cannot be represented as having a specific location. It's in a superposition, dispersed across space, and has a chance to be in all possible states. "It's neither here nor there," says Gerstein. It's described mathematically by a wave function with a probability amplitude. When the electron is measured, the wave function 'collapses' and the electron is localized.

The oddness of quantum theory is illustrated by physicist Erwin Schrödinger's classic but rather gruesome thought experiment. A cat is placed in a box along with a radioactive sample that has a $50 \%$ chance of emitting a particle, triggering the release of poison and killing the cat. Until one looks inside the box, the cat is either dead or alive. But according to quantum mechanics, the cat is both alive and dead; it's in all the states it could possible occupy. This changes only when the observer 


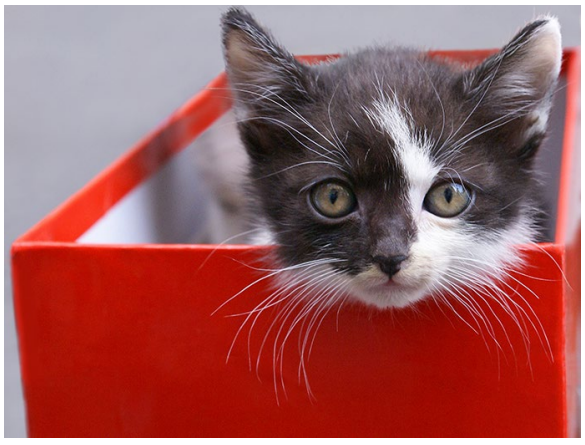

The oddness of quantum theory is illustrated by Erwin Schrödinger's thought experiment. A cat is in a box along with an apparatus that has a $50 \%$ chance of killing the cat. According to quantum theory, until an observer checks, the cat is both alive and dead. Credit: D. Todorovic/Getty

checks on the cat. The thought experiment is about subatomic particles, which unlike larger objects have variable states and can be entangled-linked to one another, even across great distances. In an entangled pair, one particle can reveal properties of the other. This is part of the Einstein-PodolskyRosen paradox ${ }^{8}$. As Gerstein explains, when two such particles are separated, measuring one fixes the measurement results on the second. "This may seem like spooky action at a distance," he says. It's akin to a separated pair of gloves. Seeing the right-handed one immediately tells you the other is the glove for the left.

Quantum computers perform calculations with qubits, which are the basic units of quantum computers. In systems with multiple qubits, qubits can become entangled. Due to quantum entanglement, any operation performed on one of the qubits will affect the collective state of all in the qubit group. Deane says it's a mathematical fact that "without quantum entanglement, no quantum algorithm can outperform a classical one."

Gerstein admits that, even for someone like him with physics training, much is mystifying about quantum mechanics. The shorthand is, he says, that the wave function is "everywhere." Qubits can possess multiple states at the same time. Computing with qubits is as if all operations in a computational workflow are performed at once. It's similar to a "massive amount of parallelism," he says. At the calculation's conclusion, the qubits' variable states collapse into an answer.

As a fast arithmetic machine, says Mulligan, a classical computer solves many problems, but it's bad at entire problem classes, such as when a problem's solution is to select the best solution among "zillions of options." A classical computer solves that by iteration. It trucks through possibilities one by one, scores each one and returns the best-scoring one. Computers can easily consider a thousand, a million, or sometimes even a billion possibilities, "but when you get into the trillions or the quadrillions or the quintillions, you start to get past the realm where even a fast computer can consider all the possibilities," he says. "That's where quantum computing is so cool; unlike a classical computer, which can only consider one possibility at a time, a quantum computer can exist in many states simultaneously-effectively, considering many possibilities at once." Classical digital bits have designations of 1 and 0 , whereas qubits have states. The number of states corresponds to the number of possibilities. This number doubles with every qubit added to a system, which offers "the prospect of considering truly astronomical numbers of possibilities when performing hard search problems." Biology is replete with those tough search problems.

Gerstein laughs as he says that he does not "super-understand" all of quantum computing's intricacies. "But it does work." Code-breaking is one application area for quantum computing, he says. In some types of attacks a quantum computer can find passwords by trying all words. It's a "brute force" way to find the password, he says. There's government interest in such capabilities. This might seem far afield from biomedicine, he says, but plenty of basic biomedical researchers, not just clinicians, need to protect the patient data they use. He hears colleagues in cryptography and computer security describe how their computational process has been "quantum-hardened," which means it is more able to withstand a quantum attack. Such concepts could find their way into biomedical research, he says.

Labs performing structural analysis might consider quantum computing. Assessing 'molecular jiggle' usually involves Newtonian mechanics, says Gerstein, but quantum mechanics helps with studying molecular bond creation and breaking. He sees labs experimenting with hybrid workflows that mix classical and quantum calculations. A lab might model the "inside of an enzyme," and some of those calculations could have a quantum component. In the future, scientists might run $80 \%$ or $90 \%$ of a workflow on classical computers and then, for the sake of efficiency, run some calculations on a quantum computer. Protein folding, which has "monster calculations," would be one area where this applies, he says. Drug design and protein design are others.

\section{Quantum peptide design}

In drug design, researchers face countless ways in which atoms in a drug molecule can be arranged, and they seek the one best suited to the particular function their drug is to perform. "If you want to predict the structure that a protein will fold into, you have to find the lowest-energy conformation of a chain of amino acids from the zillions of possible conformations that that chain can twist into," says Mulligan. Classical computers hit a wall quickly, "but the hope is that quantum computers could help us to push far past that limit," he says. "Someday, anyways-right now, current-generation quantum computers are still pretty limited." Mulligan and colleagues at several institutions and companies mapped a protein design problem to a quantum computer. To design peptides they enabled the protein design software Rosetta-in particular, its Packer moduleon D-Wave Systems quantum hardware. Protein design involves optimizing rotamers, which are the positions and conformations of a protein's side chains. That's a hard computational search problem given that such design means trying out and assessing many positions per rotamer. Rosetta runs on classical computers, simulating the interactions of atoms and molecules, and contains algorithms that researchers use to solve hard search problems such as protein structure prediction and sequence design. Peptides and proteins have a backbone of amino acids, with side chains hanging off the backbone. Each type of amino acid has a different side chain, and most side chain types can take on different conformations. The design challenge is, says Mulligan, given a fixed peptide or protein backbone that one wants to stabilize with a particular choice of amino acid sequence, to choose one amino acid rotamer at each position such that the energy is at a minimum.

Even if there is only one conformation for each of the 20 amino acid types, for a 100 -amino-acid protein that would mean 20 to the power of 100 possible sequences for that protein. "In comparison, there are about 10 to the power of 80 atoms in the universe," says Mulligan. The added complication is that there isn't just one rotamer per amino acid type, but several of them. After all, side chains are flexible. "This is what makes it a very hard problem," he says. Exhaustive enumeration on classical computers won't solve it. Typically, scientists apply heuristics such as Monte Carlo methods for such problems, which perform a semirandom search of a small subset of the possible sequences to progressively look for better and better possibilities. All these deliver pretty good solutions; they 
are not guaranteed to be the best ones, says Mulligan. With quantum computers, there is the prospect of considering all possible combinations of rotamers, as an initial equal superposition of all possible strings of $1 \mathrm{~s}$ and 0 s. One can shift the probability distribution of states "until we're overwhelmingly likely to observe a string of $1 \mathrm{~s}$ and 0 s representing the best combination of rotamers out of all of them."

The scientists worked out how to map the sequence design part to the $\mathrm{D}$-Wave, and first ran it on the 2000Q system, which has 2,000 qubits. Using that system, the team successfully designed peptides, made and characterized some of them in the wet lab, and confirmed they actually fold into the structures designed on the quantum hardware. More recently, says Mulligan, the team has used D-Wave's newer Advantage system, which has 5,000 qubits. And the team is adapting the sequence design algorithm for gate-based quantum computers such as those IBM, Honeywell and others are developing, and which are unlike the quantum annealers such as the D-Wave. Packer is the Rosetta module that addresses rotamer optimization with Monte Carlo probability assessment methods. The scientists built QPacker, a quantum version of Packer. They divided up the design tasks: a classical precalculation on an ordinary computer to calculate the energies of individual rotamers and pairs of potential interacting rotamers. "That requires fast arithmetic, which classical computers are good at," he says. The quantum algorithm did its calculations on the D-Wave to solve the hard combinatorial problem of searching the vast set of all possible combinations of rotamers and pulling out the best one, he says. In this setup both types of hardware "are doing what they do best, and avoiding the stuff that they do worst," he says.

\section{Quantum hardware}

$\mathrm{D}$-Wave and other companies have made quantum computers that use, as qubits, electrons with spins up and down to represent $1 \mathrm{~s}$ and $0 \mathrm{~s}$. In the case of the $\mathrm{D}$-Wave, computing operations involve manipulating electrons with magnetic fields, says Mulligan. But electrons and magnetic fields aren't fundamental to quantum computing, he says. Honeywell, IonQ and other companies are exploring quantum computers that use completely different physical systems that allow you to do the same things. It's just like how a "steam-and-valve classical computer could do the same logical operations that an electronic wire-and-transistor classical computer can do," he says. Quantum companies have been working on ways to



Peptide design involves searching through many possibilities, which quantum computing does well. For this task, Vikram Mulligan and team built a quantum version of the Packer module from the Rosetta package. Credit: V. Mulligan, Flatiron Inst.; T. Phillips, Springer Nature

build qubits that involve manipulating the state of atoms, the spin of electrons or the vibration of ions, to name a few.

Digital bits are either 1 or 0 ; qubits can be both 1 and 0 : they can have a superposition of states. Quantum algorithms perform calculations as operations on these states. "It's hard to isolate these quantum states," says Gerstein. That's why, for example, some hardware implementations in quantum computing involve superconducting materials to keep circuits from interfering with one another. These circuits are unlike traditional chips with transistors etched onto silicon. But quantum computers have their own hardware challenges as companies explore many types of implementations. Some architectures use electrons that are manipulated by magnetic fields. Among the issues in hardware design, says Gerstein, is the number of qubits one can assemble and how to set up quantum RAM.

Some hardware involves superconducting environments that need to be supercooled; others, such as IonQ's trapped ion technology, operate at room temperature. "We don't know five years from now what the leading technology will be," say IonQ's Ruffner. And there may be several. Hardware for quantum computing is still evolving, says Senthil, but some labs implement quantum machine learning algorithms on existing quantum hardware systems. She points to an NIMH-funded project ${ }^{9}$ by the University of Virginia School of Medicine's Stefan Bekiranov, Kunal Kathuria and colleagues to analyze single-cell neuronal genomic copy number variation in samples. The team developed classifiers and used quantum computing to compare synthetic data about brain tissue from people with Alzheimer's disease and those without. Quantum computing delivers "an exponential speedup" in a core calculation, says Bekiranov. The team used the IBM Q simulator and IBM 5-qubit and 14-qubit quantum computers. As quantum computing power and fidelity advances, they plan to use real data.

\section{Algorithms go quantum}

Algorithm developers and users will want to bear in mind, says Deane, "that quantum computers are likely to only confer an advantage in terms of speed for only a limited number of tasks." And, she says, "you can't just port a classical algorithm onto a quantum computer."

On classical computers, says Gerstein, algorithms used in biomedical research map sequence reads, impute genomic variants and analyze behavior, among other things. Particularly in neuroscience, when characterizing molecules, cells, tissue, organisms and behavior, calculations are performed within and across scales. Big-data challenges such as population-scale analyses might also be amenable to calculations performed on a quantum computer. Various types of machine-learning approachesBoltzmann machines, variational autoencoders, neural networks-can be used for analyses on those different scales. He and others explore how to make algorithms 'quantum'-for example, turn a Boltzmann machine into a quantum Boltzmann machine. The Boltzmann machine is a kind of neural network that computes an 'energy function' as it tackles problems, he says. It's used, for example, to perform calculations that are part of single-cell analysis or to impute epigenomic modifications. With a Boltzmann machine, a user is frequently searching for the lowest energy state, "and a quantum calculation can help you with that," he says. A quantum Boltzmann machine could be useful in many computational biology contexts.

In Hunter's view, given how crucial hidden Markov models are to sequence analysis, quantum extensions of them, both in terms of new classes of model and the quantum speedups they provide, could be practical soon. "A practical quantum variational autoencoder or other machine learning quantum speedups would be broadly important, including in biomedical research," he says.

Overall, says Mulligan, "making a quantum version of an algorithm is tricky." There are some standard quantum algorithms that have been devised for use on quantum computers, such as Grover's 
algorithm, Shor's algorithm and others. If an algorithm can be expressed in terms of those, it may be easier to convert. Although quantum computers will in theory one day be able to perform any operation that a classical computer can, plus be able to do hard search problems, "it's very likely that they will always be a thousand to a million times slower at basic arithmetic than a classical computer is," he says. Thus, there's no advantage to using a quantum computer to do payroll, or to render a CGI movie, or to do many of the other things that are already done well on classical computers.

It's the hard search problems where there's likely to be an advantage, says Mulligan. "Even there, it's still a challenge to come up with ways to convert a classical algorithm to a quantum one." In many ways, he says, the team got lucky with Packer and QPacker because the problem that Rosetta was trying to solve was one involving hard search and it was structured in a way geared toward ones the D-Wave can solve. The D-Wave system, he says, is not a general-purpose quantum computer; it's geared toward solving one type of problem. Among the collaborators in the QPacker project are colleagues at Menten AI, a company Mulligan co-founded, although he himself is full-time at Flatiron. Menten is keen on using quantum computing for drug design, says Mulligan, but they use other approaches, too, such as machine learning "on trusty old classical computers" and physical simulations with software like Rosetta. For the drugs they work on, says Mulligan, the Menten teams seek to move beyond traditional small-molecule drugs to peptide therapeutics, which he views as an underdeveloped but very powerful class of drugs. As they watch quantum computing develop, they will explore how to use it to its full potential, but in the meantime continue to do plenty with classical computers.

\section{Quantum languages}

Scientists interested in quantum computing have many options. At IonQ, Ruffner handles inquiries from potential collaborators and users in many areas, but she has a penchant for biology. She used to be part of the IBM Quantum team and remembers a phone call from a scientist asking for "translation software" so the lab could run its algorithm and software on a quantum computer. "It doesn't quite work like that right now," she recalls telling the researcher. "Essentially you have to reprogram it for a quantum computer, and it does take a specific set of skills to do that."

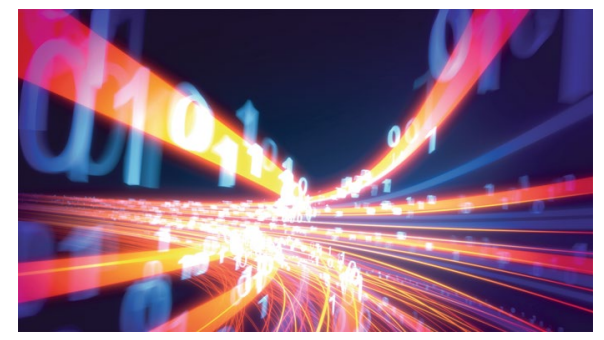

Classical computers that work with digital bits are speedy. But for some types of calculations, quantum computers can be blazingly fast. Credit: Y. Chino/Getty

Some companies have developed open source languages for programming quantum computers. Google has Cirq, Microsoft has Q\# and IBM has Qiskit. IonQ decided to not develop yet another language, says Ruffner: "we feel there is already too many of them out there." IonQ users can any of these languages on IonQ computers, which one can access through the company's cloud. Quantum computers are also accessible through Amazon Web Services, Microsoft Azure and others. She points to the many courses researchers might explore-for example, MIT OpenCourseWare and edX offerings. "There's a little bit of energy that you have to expend to get into quantum computing, she says. "Once you're there, then it's your own creativity on problem solving." The effort might make something that was computationally cumbersome or perhaps even intractable become a readily completed task. Ruffner recalls speaking with a biologist working on a genome-wide association study (GWAS) that was going to take a year and a half of computing time. Quantum computing can help in such instances, she says. Neuroscience is another area that could benefit from quantum computing. "I do believe not enough biologists have taken a look at quantum computing," she says. "It would be great to speed up GWAS, but it would also be great if you could think of something new and different that you've always wanted to do but the computing was just too complex." That might be in drug design, multimodal data analysis in genomics, or any of various problems in neuroscience.

Ruffner has also developed a networking chapter for women in quantum computing within an organization she and others set up called One Quantum.

\section{Quantum outlook}

"Quantum computing is finally starting to become practically useful," says Hunter, and that is going to accelerate with further technical progress. One also needs to be cautious, he says, given that many attractive theoretical ideas such as those that require quantum RAM "may never become practical."

"Quantum mechanics is unquestionably one of the most important theories in science," says Gerstein. And systems that harness quantum mechanics will be of interest across the sciences, including in biomedical research. "People are going to want to take advantage of those properties to do calculations in the future," he says.

"As for how quantum computing will work in the long run, it's still anyone's guess," says Mulligan. The hardware will probably always be bulkier and more expensive than classical computers, but that depends on which physical implementation ends up winning out. It might be electrons, trapped ions, photons or something else. For the immediate future, he says, quantum computing will likely be a computing service: users upload problems to a cloud-based quantum computing provider, they run a calculation on a quantum processor and use it alongside a classical computer that is perhaps also cloud-based, and users download results. This is similar to using cloud-based computing resources now available in high-performance computing. "But whether we'll have workplaces with their own dedicated quantum computers someday or not is hard to say," he says. "I kind of hope that we do."

Vivien Marx ${ }^{\bowtie}$
Nature Methods.
$\bigotimes_{e-m a i l: \text { v.marx@us.nature.com }}$

Published online: 23 June 2021

https://doi.org/10.1038/s41592-021-01199-Z

References

1. Aronson, S. Shtetl-Optimized https://www.scottaaronson.com/ blog/?p=5387 (2021)

2. Outeiral, C. et al. WIREs Comput. Mol. Sci. https://doi.org/ 10.1002/wcms.1481 (2020).

3. Mulligan, V. K. Preprint at bioRxiv https://doi.org/10.1101/752485 (2020).

4. Emani, P. et al. Nat. Methods https://doi.org/10.1038/s41592-02001004-3 (2021)

5. Li, R. Y. et al. Patterns https://doi.org/10.1016/j.patter.2021. 100246 (2021).

6. Arute, F. et al. Nature 574, 505-510 (2019).

7. Preskill, J. Quanta Magazine (2 October 2019); https://www.quantamagazine.org/ john-preskill-explains-quantum-supremacy-20191002/

8. Einstein, A., Podolsky, B. \& Rosen, N. Phys. Rev. 47, 777 (1935).

9. Kathuria, K., Ratan, A., McConnell, M. \& Bekiranov, S. Quantum Mach. Intell 2, 1-26 (2020). 